\title{
ANALYSIS OF JAPANESE FOLDING FAN'S CHARACTERISTIC STRUCTURE AND TRADITIONAL TECHNICAL SKILL
}

\author{
FUJIKO ABE ${ }^{1}$, YOSHIFUMI OHBUCHI ${ }^{1}$, TSUTAO KATAYAMA ${ }^{2} \&$ HIDETOSHI SAKAMOTO ${ }^{2}$ \\ ${ }^{1}$ Kumamoto University, Japan \\ ${ }^{2}$ Doshisha University, Japan
}

\begin{abstract}
Japanese folding fans were born during the Heian Period (794-1185) and have been used in every situation. Many Japanese fans are used widely at present as a portable fan, a tool of Japanese dance and tea ceremony. The making of folding fans in Japan has a history of 1,000 years or more and the production technology of the Japanese fan has been followed up until now. This research has aimed to clarify the characteristics of the traditional Japanese fan through the material of fan components, the fan-making process and the traditional production skill of a Japanese fan.

Keywords: Japanese folding fan, natural material, traditional skill, reproduction, concave-convex effect.
\end{abstract}

\section{INTRODUCTION}

The making of folding fans in Japan has a history of 1,000 years or more and the production technology of the Japanese fan has been followed up to now [1]. The function of a folded and portable fan that was an invention of Japan, has spread all over the world, and its materials were changing in various countries. The drawing and production technique of a fan was polished up by the painters who represent Japan in the Edo era (1603-1868) [2], [3]. Moreover, the predecessors' wisdom about the production technique and a lot of the processing technologies are condensed by fan-making craftsmen [4]. In previous papers [5][7], the quantitative evaluation of the shaped form, the historical transition and the workstudy program for understanding the Japanese folding fan were shown.

The material and structural characteristics of a Japanese fan are examined in detail in this research. Moreover, the function and the artistry arising from those characteristics are considered.

\section{MATERIALS AND MANUFACTURING PROCESS}

\subsection{Materials of Japanese traditional fan}

A traditional Japanese folding fan consist of (a) thin bamboo bones and (b) fan-shaped Japanese handmade paper "Washi" which was processed for the fan. "Washi" is a cellulose fiber reinforced paper and the fiber orientation is mainly at right angles to the folding direction as shown in Fig. 1(b). Additionally, "Washi" is a layer structure shown in Fig. 1(c). Fig. 2 shows the assembled bamboo bones and the part's name. The thickness of "Washi" for a folding fan is about $0.13-0.16 \mathrm{~mm}$.

\subsection{Manufacturing process}

The manufacturing process is shown in Fig. 3.

In this manufacturing process, it is difficult to insert the middle bone "Nakabone" into Washi (Japanese paper) in D-stage and E-stage. Here, the sebum has an important role. The 
I

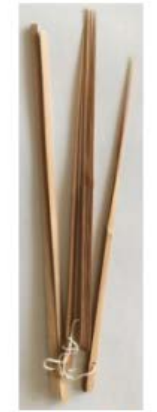

(a) bamboo bone



(b) Japanese paper "Washi" (fiber distribution)
Layer structure

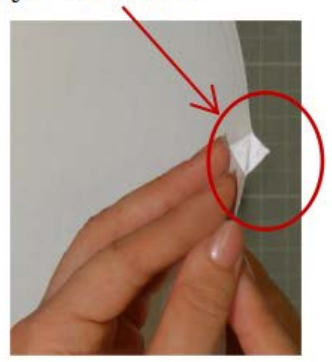

(c) layer structure"Washi"

Figure 1: Materials of traditional Japanese folding fan.



Figure 2: Assembled bamboo bones.

craftsman puts up to the bamboo bone of the fan by touching his/her scalp as shown in Fig. 4 in order to reduce the insert friction of paper and prevent the tearing of paper. After this operation, a small route in Japanese paper for insert has to be made with the bamboo bone in advance before inserting "Nakabone" into paper ("Nakazashi" process) and the inserting process is then carried out. Bamboo bones "Nakabone" are inserted into the direction of thickness of the Washi because of the layer structure shown in Fig. 1(c). The "Nakabone" insertion structure of Japanese folding fan is greatly different from that of fans of other countries.

\section{STRUCTURAL AND ARTISTIC CHARACTERISTICS OF JAPANESE FOLDING FANS}

In this section, Japanese folding fan's structural and artistic characteristics are shown in comparison with those of other countries' fans [8]. Fig. 5 shows some typical examples of European style folding fans [8]. The themes from literature and daily life are painted on the fan face; in Fig. 5(a), 5(b) and 5(c) is a fan with lace. Fig. 5(d) shows a jour fan which is called "Decoup" and Fig. 5(e) is a fan with slits which is called "Cabriot". 


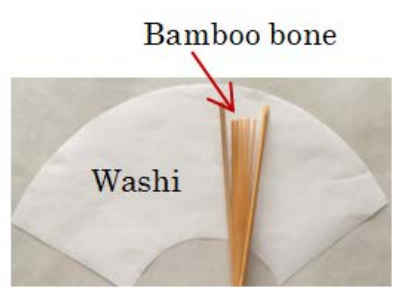

(A) materials

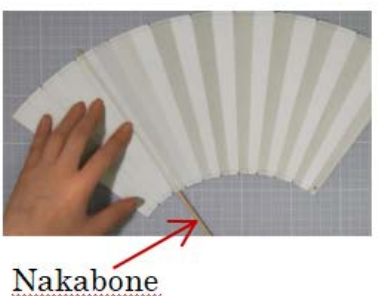

D) provisional insert process of Nakabone
Folding paper guide

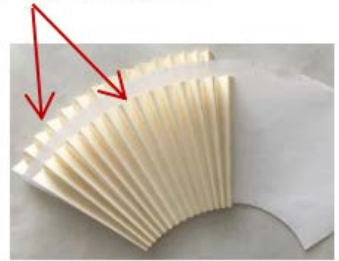

(B) folding process

(C) cut

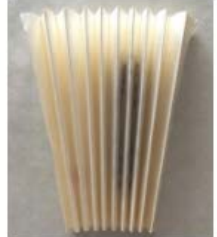

parts

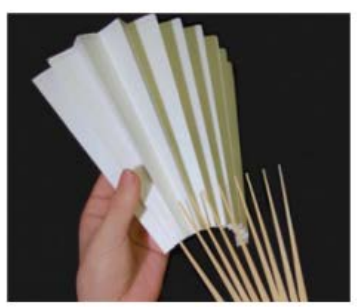

(E) final insert process of Nakabone

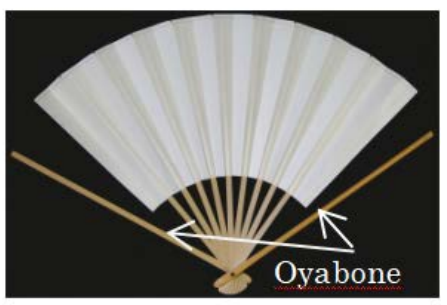

(F) gluing process

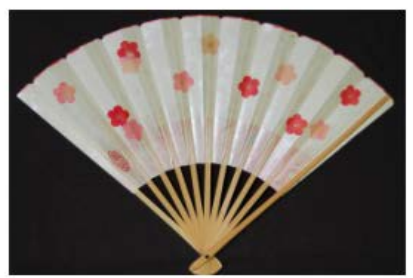

(G) finished folding fan

Figure 3: Manufacturing process of folding fan. (A) Arrangement of bamboo bones and the sector of Japanese paper "Washi"; (B) Tracing the folding lines on the fanshaped paper with a folding guide, which is a pair of accordion folded paper guide; (C) Cutting the top and bottom of the fan-shaped paper; (D) Dispreading the folded fan-shaped paper, and inserting the thinner middle bones (Nakabone) into the middle of the dispread paper ("Nakazashi": provisional insert process); (E) Insertion of the starch-pasted thinner middle bones into the dispread paper; (F) Gluing up the dispread paper and the thinner middle bones; and (G) Adhering two thick bamboo bones (Oyabone) to both ends of the dispread paper.

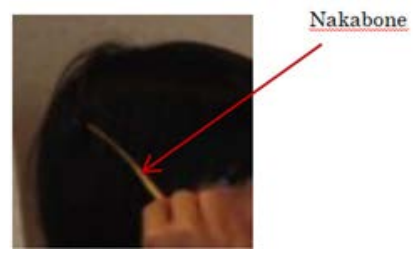

Figure 4: Putting up to the sebum on bamboo bone "Nagabone". 


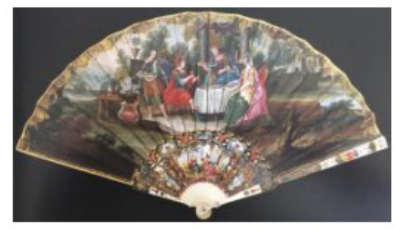

(a)

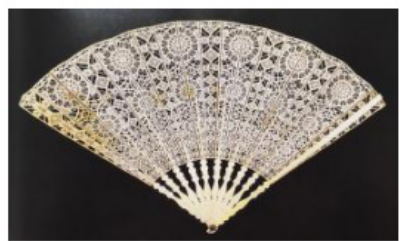

(d)

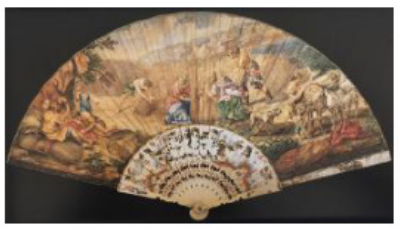

(b)

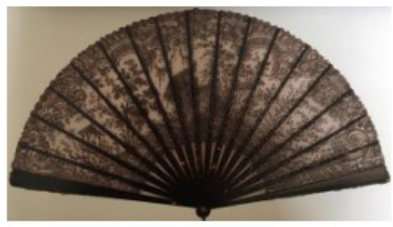

(c)

Figure 5: Examples of western style folding fan. (a), (b) and (c) Fans with lace; (d) A jour fan which is called "Decoup"; and (e) A fan with slits which is called "Cabriot".

First, the structural characteristics of the fan are considered. In European style fans, the fan consists of the wooden board or the ivory board for the folding fan's bone and the cloth shown in Fig. 5. The fan made only from a wooden board and the board of the ivory is common. In this case, the waving is given to the fan's bone boards. Therefore, these are heavy and bulky in comparison with those of a Japanese fan. As an arbitrary opening angle cannot be adjusted, the fan opens completely and is used.

In contrast to these, a Japanese folding fan has the folding structure of Washi shown in Fig. 6(a). With this structure, it can be adjusted to an arbitrary opening angle. The Japanese folding fan has large strength because the folding structure produces the 3-D structure effect, though thin bamboo bones are used. As a result, the airflow can be effectively created. Fig. 6(b) shows another portable fan "Uchiwa". This has the same function as a folding fan and is made of the same materials. But, this is a more flexible than the folding fan because of its two dimensional structure and it is inconvenient to carry as it cannot fold up.

\section{Folding}

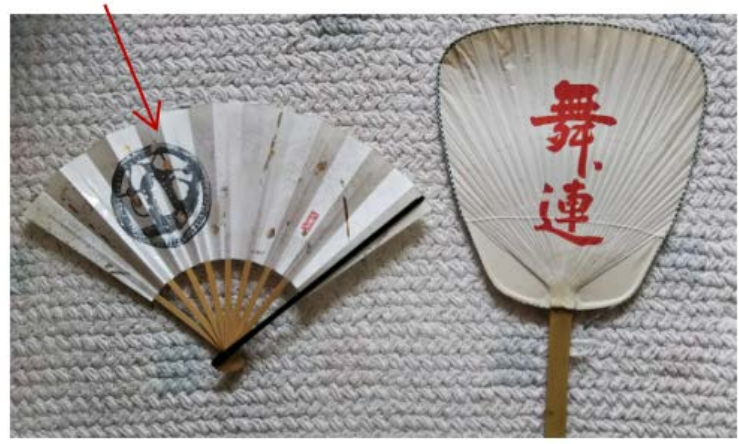

(a) folding fan

(b) Uchiwa

Figure 6: Japanese folding fan and 2D-portable fan "Uchiwa". 
Next, the artistic characteristics of the fan is considered. The western style folding fan is used while opened completely. Therefore, the pictures drawn on the fan face are in two dimensions, and it is the same as a usual painting. However, a Japanese fan has the folding as shown in Fig. 6(a). Therefore, the picture drawn on the fan face appears different from the usual picture painted in two dimensions. The geometrical shape of a two dimensional picture on the fan face changes by the folding effect shown in Fig. 7. Moreover, the distortion depends on the fan shape. Even if the fan shaped paper sizes are the same, the distance $l$ from joint pin "Kaname" to drawing objects give influences in the distortion of the painted shape as shown in Fig. 8. As just described, the distortion of them is very complicated and depends on the distances [9], [10].

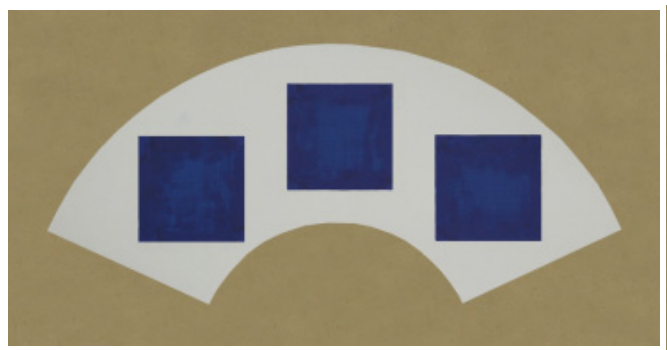

(a)

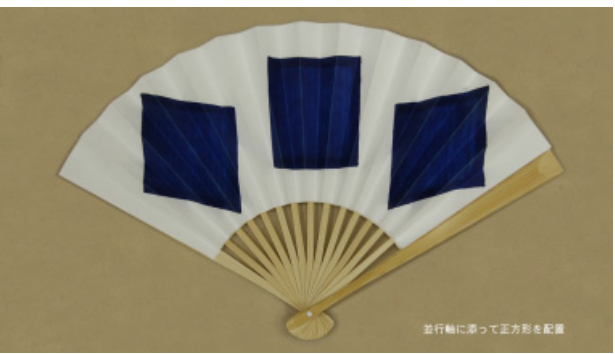

(b)

Figure 7: Geometry shape of picture change. (a) 2-D picture; and (b) Folding fan picture.

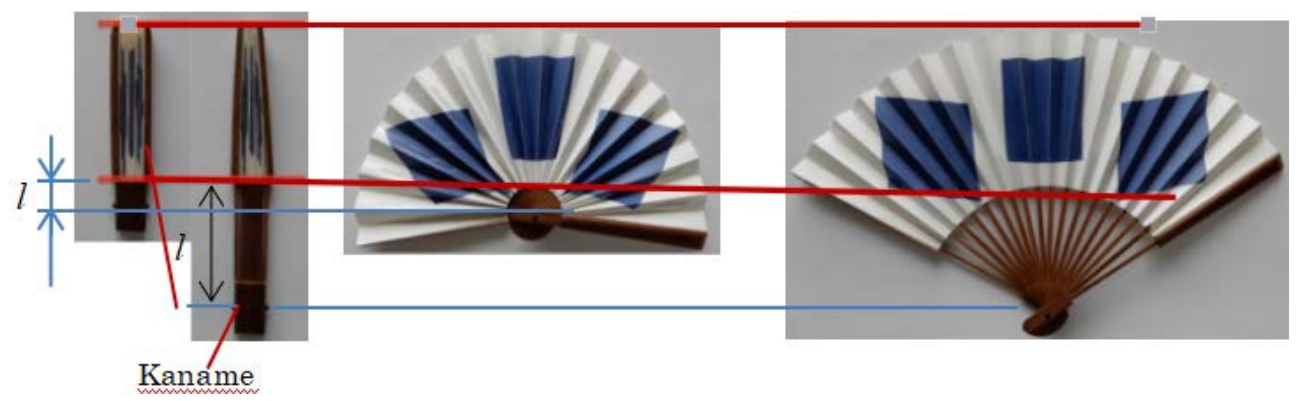

Figure 8: Distance dependency of distortion shape in a folding fan (in the case of a same fan shaped paper).

When the painter draws the folding fan picture, it is important to understand the distortion mechanism. The folding fan picture becomes a picture of a correct composition when the folding fan is opened to final position. An example of the traditional Japanese fan is shown in Fig. 9.

As previously stated, the pictures or the images drawn on the folding fan face are deformed in a complex manner according to the size of the structure. So, an image transformation program was created for mathematically analyzing the deformation. The original image and the experimental and analytical results are shown in Figs 10 and 11, respectively. It is understood that the difference of the distance from "Kaname" greatly influences the distribution of fan face distortion as can be deduced from Fig. 11. 


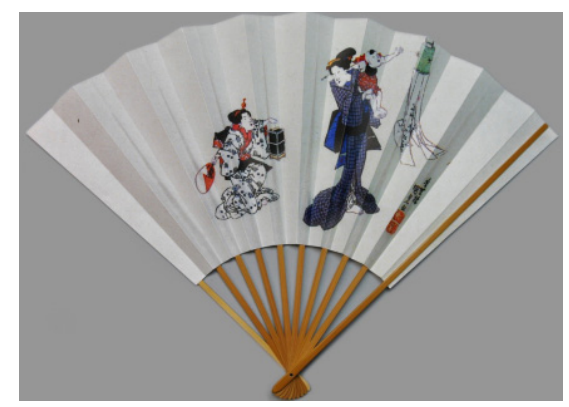

Figure 9: An example of the traditional Japanese fan.



Figure 10: Original image (2-D painting).

The simulation can represent a nearly accurate behavior. This result makes it easier for a successor to understand how the images would be transformed. Hence, they would acquire the technique to find the best way to draw pictures, taking the deformation into consideration. Referring to the result, the successors would be able to understand the traditional Japanese fan deeply.

As another characteristic, there is the concave-convex illusion effect by the folding. The perspective is caused by arranging the drawing object on the mountain segments or valley segments of the folding fan. Furthermore, the Japanese folding fan can be arranged by controlling its opening angle.

\section{SUMMARY}

Traditional Japanese folding fans consist of thin bamboo bones and "Washi", which is a cellulose fiber reinforced paper, and the fiber orientation being mainly at right angles to folding direction. The 3-dimensional structure of folding fan given by the folding produces the concave and convex illusion effect and the perspective effect. The Japanese fan creates various artistic impressions by changing the opening angle of the folding fan. The complex distortion of the pictures or the images drawn on the folding fan face, was clarified by the proposed image conversion program. 




(a-1)

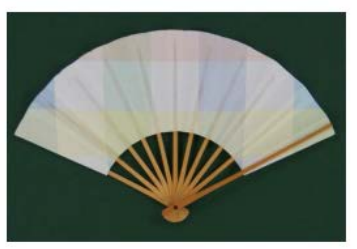

(a-2)

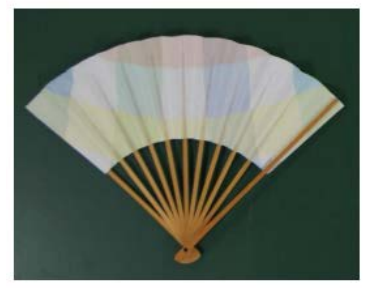

(a-3)



(b-1)

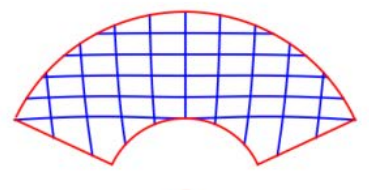

(b-2)

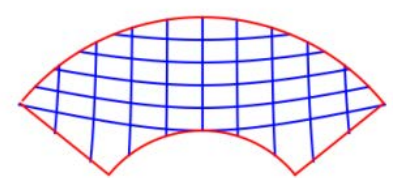

(b-3)

Figure 11: Comparison between experiment and simulation results. (a) Experiment; and (b) Simulation.

\section{REFERENCES}

[1] Nakamura, N., Senmen-ga and Ougi-E, Kawahara Publishing Co., 1969.

[2] Kobayashi, T., Semmen-ga. Japanese Art, 32, 1993.

[3] Misuo, M., Summary of Semmen-ga, Kourin Publishing Co., 1981

[4] Ōta Memorial Museum of Art, www.ukiyoe-ota-muse.jp/exhibition/2011. Accessed on: 20 Dec. 2018.

[5] Abe, F., Ohbuchi, Y. \& Sakamoto, H., Quantitative evaluation by measurement for production technology and reproduction of traditional folding fan. Proceedings of the 13th International Symposium on Advanced Science and Technology in Experimental Mechanics, A19040, pp. 1-5, 2018.

[6] Abe, F., Ohbuchi, Y. \& Sakamoto, H., Transitions of Japanese folding fan for export in Meiji era. Proceedings of Society for Design and Art Fusing with Science and Technology, pp. 12-17, 2018.

[7] Abe, F., Ohbuchi, Y. \& Sakamoto, H., Learning about Japanese traditional technique by experiencing how to make a "folding fan". Proceedings of the 7th Asian Conference on Engineering, pp. 15-16, 2018.

[8] Bennet, G.A., Unfolding Beauty: The Art of the Fan. The Collection of Ester Oldmam and the Museum of Fine Arts, Thames and Hudson, USA, 1998. 
PI-102 Computational Methods and Experimental Measurements XIX

[9] Minde, K., Distortion in pictorial scenes on Japanese fans. Journal of Graphic Science of Japan, 43(2), pp. 3-9, 2007.

[10] Koyama, S., Structure of Semmen-ga. Journal of Graphic Science of Japan, 33, pp. 127-132, 1999. 